\title{
Diversification of the drug discovery process
}

\author{
Allen Krantz
}

Drug discovery and development are highrisk, high-stakes ventures with long and costly timelines. It takes 7 to 12 years to bring a drug to market, at a cost of up to $\$ 300$ million. A major financial impediment to commercializing drugs is the 1 -in12 odds of surviving phase 1 clinical trials.

Despite these very poor odds and long timelines, the prospect of generating billion dollar sales per annum for at least a decade provides pharmaceutical companies with powerful incentives. To increase the chances of producing successful drugs, pharmaceutical companies have begun to diversify the process of drug discovery by seeking out technologies that are likely to produce the broadest range of drug candidates.

Consequently, the field of drug discovery is being rapidly transformed by technological advances that profoundly affect the modus operandi of researchers engaged in lead discovery and optimization. The impact of the "foundation trinity" (combinatorial chemistry, genomics, and highthroughput screening) that has informed diversity-based drug discovery has been described recently by Dale Pfost in a stimulating commentary (Nat. Biotechnol. 16:313, April 1998). These methods have been embraced by the major pharmaceutical companies because of the speed with which they allow new molecules to be generated and screened against validated targets.

Indeed, more targets, more compounds, and more published patents are important quantitative indicators of progress. Yet although the costs for generating and screening new compounds have been substantially reduced, and the process by which new drug candidates are identified has been markedly accelerated, it is not clear whether the central qualitative issue of reducing the failure rate of drugs in clinical trials is being addressed by these quantitative changes.

The notion of minimizing risk by diversifying the structural features of drug candidates is a very practical one. In any highrisk venture, hedges against risk are important strategic components. After all, drugs fail for many reasons, essentially all of which have to do with their molecular structures. Drug resistance, allergic and immune reactions, adverse drug interac-

Allen Krantz is president and CEO at Bullet Therapeutics in San Mateo, CA (akrantz@ix.netcom.com). tions, and problems related to bioavailability encompassing absorption, distribution, metabolism, and excretion are not uncommon. Drug toxicity is often a result of interference with a protein whose function is undesirably affected because it possesses a site that is structurally similar to the intended target. As drugs are so vulnerable to failure from diverse sources, and neither the critical structural features of drugs (pharmacophores) nor their target protein sites are easily altered, it is important to

\section{Drug-targetable sites on} proteins pursued to date have generally been confined to single, endogenous, ligand-binding sites. To exploit site diversity, we need methods for uncovering alternate binding sites and ways of screening ensembles of molecules for their affinities toward such sites.

generate a broad range of structures for drug candidates.

It is often assumed that the issue of the structural diversity of leads is addressed by screening libraries of enormous numbers of compounds. However, the full potential for developing diverse series of drug leads and drug candidates depends not only on screening vast numbers of compounds against a validated target but also on exploiting the full panoply of mechanisms and loci available for direct attack on a specific target protein.

A clear concern is that current methods of lead discovery are likely to afford only modest improvements in diversifying risk to the extent that they produce leads to only a single site per protein. Unless earnest attempts to achieve general methods for probing the protein surface are pursued, it is reasonable to expect that most ligands will concentrate at endogenous ligand sites that have evolved over eons of time to bind natural ligands.
The topography of protein sites imposes limits on the structural diversity of drugs, in that the requirement for complementarity between ligand and protein binding site circumscribes the structural features of drugs. Thus, all drugs tightly bound to the same site will have some common structural features. Consequently, targeting a single site, either by design or in a de facto sense, is tantamount to putting all of one's eggs in one basket and probably contributes to the high failure rate of drug candidates.

Maximally diversifying the molecular structures of drug candidates requires the use of all potential binding sites to exploit site diversity. Strategies that are designed to capitalize on the possibilities for intervention along the entire protein surface have been slow to evolve, in part because the idea that site-diversity governs the structural diversity of drugs has not been widely promulgated.

The notion of protein-site targeting and the development of enabling technologies for probing the protein surface for "hot spots" is an inevitable consequence of theoretical research designed to uncover the local energy minima of ligand-protein complexes, structure-based design, and high-throughput screening. We can discern this shift in the crosscurrents of activity in structural biology. The concept of proteins as molecular machines should stimulate strategies designed to disrupt the "machinery" at various critical points. Protein architecture, with its loops, flaps, hinges, and channels, provides ample targets for broad-based inhibition strategies. Indeed, there are now plenty of examples-noncompetitive receptor antagonists, noncompetitive inhibitors, and certain covalent inactivators-to show that ligands do not have to occupy the same site as the natural ligand in order to be inhibitory.

Drug discovery and drug development are numbers games in which the odds are heavily weighted against success. Drug-targetable sites on proteins pursued to date have generally been confined to single, endogenous, ligand-binding sites. To exploit site diversity, we need methods for uncovering alternate binding sites and ways of screening ensembles of molecules for their affinities toward such sites. Sensitive probes, capable of revealing novel binding sites, offer a basis for diversifying risk by significantly expanding opportunities for blocking protein function. 